\title{
A novel method for preparation of hollow and solid carbon spheres
}

\author{
BOYANG LIU, DECHANG JIA*, JIANCUN RAO, QIANGCHANG MENG and \\ YINGFENG SHAO \\ Institute for Advanced Ceramics, Harbin Institute of Technology, Harbin 150001, China
}

MS received 12 December 2007; revised 24 February 2008

\begin{abstract}
Hollow and solid carbon spheres were prepared by the reaction of ferrocene and ammonium carbonate in a sealed quartz tube at $500^{\circ} \mathrm{C}$. The morphology and microstructure of the product were characterized by X-ray diffraction, Raman spectroscopy, scanning electron microscopy and transmission electron microscopy. The carbon spheres are amorphous and their diameters range from $0.8-2.8 \mu \mathrm{m}$. The shell thickness of the hollow carbon spheres is not uniform and ranges from $100-180 \mathrm{~nm}$. It is suggested that ammonium carbonate is crucial for the formation of carbon spheres and its amount also influences the morphology of the product. The method may be suitable for large scale preparation of carbon spheres.
\end{abstract}

Keywords. Hollow carbon spheres; solid carbon spheres; ferrocene.

\section{Introduction}

Carbon spheres, including hollow carbon spheres (HCSs) and solid carbon spheres (SCSs), exhibit excellent properties such as outstanding biocompatibility (Bokros 1977), high chemical inertness, high specific surface area, thermal insulation, low effective density and high compressive strength (Li et al 2006), which make them appropriate materials to be used in lithium-ion batteries (Lee et al 2003), fuel cells (Wen et al 2007), drug delivery, active material encapsulation (Zhong et al 2000), damping materials and composites (Zhang et al 1993) etc. For these potential applications, extensive efforts have recently been devoted to the exploration of various synthesis approaches for carbon spheres with emphasis on CVD, template assisted and solvothermal methods. Generally, the CVD method, which is popular in preparation of a diverse range of carbon nanostructures, can also be used to prepare SCSs by pyrolysis of hydrocarbons (Wang et al 2007). On the other hand, the template assisted method is usually employed to synthesize HCSs (Wang et al 2006). In brief, the templates are first coated either by pyrolytic carbon or polymers on their surfaces to obtain core-shell structures. Then the templates are subsequently removed by wet chemical etching in an appropriate solvent or calcination at elevated temperature in an inert atmosphere to create HCSs. For the solvothermal method, both HCSs and SCSs can be prepared under high pressure and at a moderate temperature for several hours in an autoclave using different reactants and solvents (Wu et al 2006; Yi et al 2007).

However, low yield and complex process are still the main factors that hinder the applications of carbon spheres.

*Author for correspondence (dcjia@hit.edu.cn)
Therefore, there is an emerging need for the cost-effective production of carbon spheres in large quantities under mild experimental conditions. In this paper, we report an efficient and low cost method to prepare HCSs and SCSs by the reaction of ferrocene and ammonium carbonate in a sealed quartz tube at $500^{\circ} \mathrm{C}$. The formation mechanism is also discussed.

\section{Experimental}

In a typical experiment, $100 \mathrm{mg}$ ferrocene and $150 \mathrm{mg}$ ammonium carbonate (a mixture of $\mathrm{NH}_{4} \mathrm{HCO}_{3}$ and $\mathrm{NH}_{2} \mathrm{COONH}_{4}$ ) were put into a quartz tube (about $30 \mathrm{ml}$ in volume) which was then sealed after pumping. Next the tube was heated in an air furnace to $500^{\circ} \mathrm{C}$ at a ramp rate of $25^{\circ} \mathrm{C} / \mathrm{min}$. After being held for $30 \mathrm{~min}$, the furnace was cooled down naturally. The as-prepared product was thoroughly washed with $\mathrm{HCl}$ solution and deionized water for several times in sequence, and finally dried in an oven at $100^{\circ} \mathrm{C}$ for $12 \mathrm{~h}$. The product was characterized by Shimadzu XRD-6000 X-ray diffractometry (XRD) with $\mathrm{CuK} \alpha$ radiation $(\lambda=1.5418 \AA)$. The morphologies of the product were recorded by Hitachi S-4700 field emission scanning electron microscopy (SEM) and Phillips Tecnai F30 transmission electron microscopy (TEM). The Raman measurement was carried out with a Jobin-Yvon LabRam Raman microscope at an excitation laser wavelength of $632.8 \mathrm{~nm}$.

\section{Results and discussion}

Figure 1a is the typical XRD pattern of the as-prepared product before washing and all the sharp diffraction peaks 
could be well indexed as $\mathrm{Fe}_{3} \mathrm{O}_{4}$ (JCPDS 19-0629). The broad peak around $2 \theta=26^{\circ}$ with low peak intensity becomes evident when the $\mathrm{Fe}_{3} \mathrm{O}_{4}$ was removed by the acid washing process, as shown in figure $1 \mathrm{~b}$. The broad peak indicates that the product is amorphous, probably due to the low synthesis temperature.

Figure 2 is the Raman spectrum of the product after washing recorded at room temperature. The prominent peaks at 1341 and $1586 \mathrm{~cm}^{-1}$ correspond to the carbon D-band and G-band vibration modes, respectively. The G-peak originates from the vibrations of $s p_{2}$-bonded carbon atoms in a two-dimensional graphite plane, while the D-peak is related to double-resonance Raman process in disordered carbon (Antunes et al 2006). The high intensity ratio of $D$ to $\mathrm{G}$ band suggests that the graphitization of the product is poor, which is consistent with the XRD pattern (figure 1b).

Figure 3a shows a SEM image of the carbon spheres without dispersion and centrifugal treatment. It is clearly

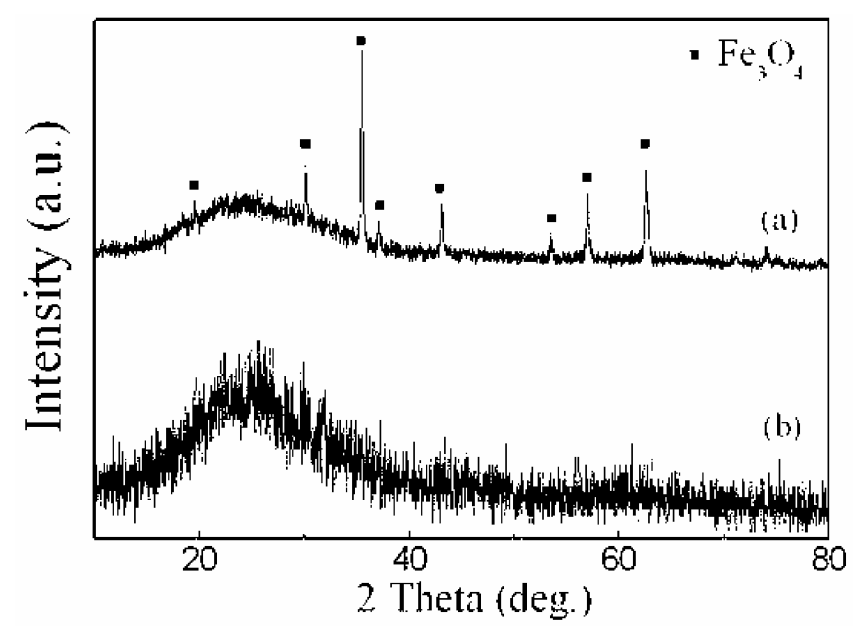

Figure 1. XRD patterns of (a) the as-prepared product in the quartz tube and (b) the product after acid washing.

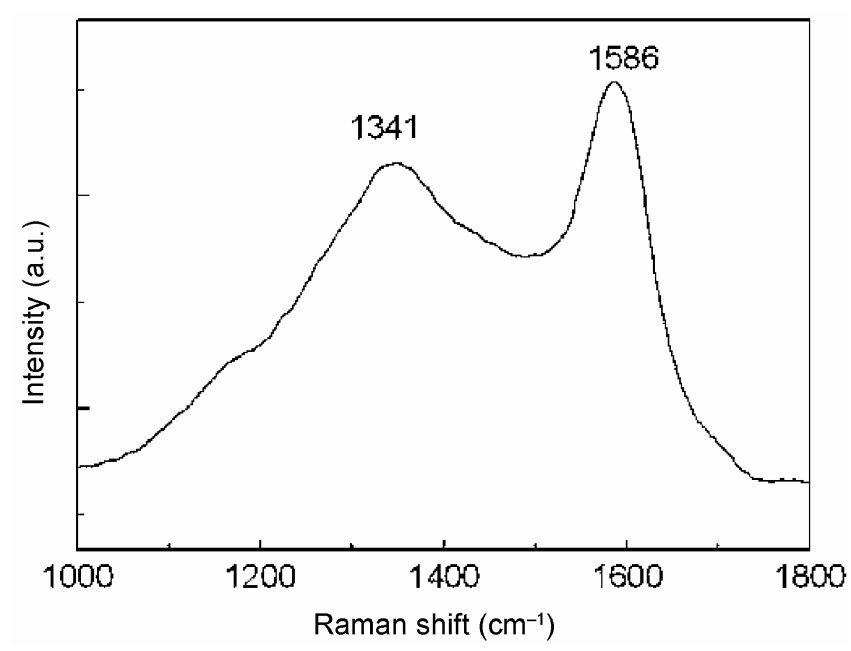

Figure 2. Raman spectrum of the product after acid washing. demonstrated that the majority of the products exhibited perfect spherical morphology. The spheres vary from $0 \cdot 8-2 \cdot 8 \mu \mathrm{m}$ in diameter with very smooth surfaces. Figure $3 \mathrm{~b}$ presents the morphology of some broken and shrivelled HCSs, suggesting that some spheres have hollow structures. From the fractured spheres, it could be assessed that the wall thickness is not uniform and ranges from 100$180 \mathrm{~nm}$.

Figure 4a shows a TEM image of a shrivelled HCS with a crack. The hollow structure could be indicated by the pale interior and dark edge. The diameter of the sphere is about $2.8 \mu \mathrm{m}$ and the thickness of the shell is uniform and about $170 \mathrm{~nm}$. Additionally, the dark spheres of $1 \mu \mathrm{m}$ in diameter could also be found, which should be SCSs, as shown in figure $4 \mathrm{c}$. Figures $4 \mathrm{~b}$ and $\mathrm{d}$ provide the high resolution TEM (HRTEM) images of the fine structure near the surface of the HCS and SCS. It is shown that both of them have amorphous structures, which is consistent with the XRD and Raman results.

In order to investigate the formation mechanism, additional experiments were further carried out. It was found that only the carbon of irregular shapes could be synthe-
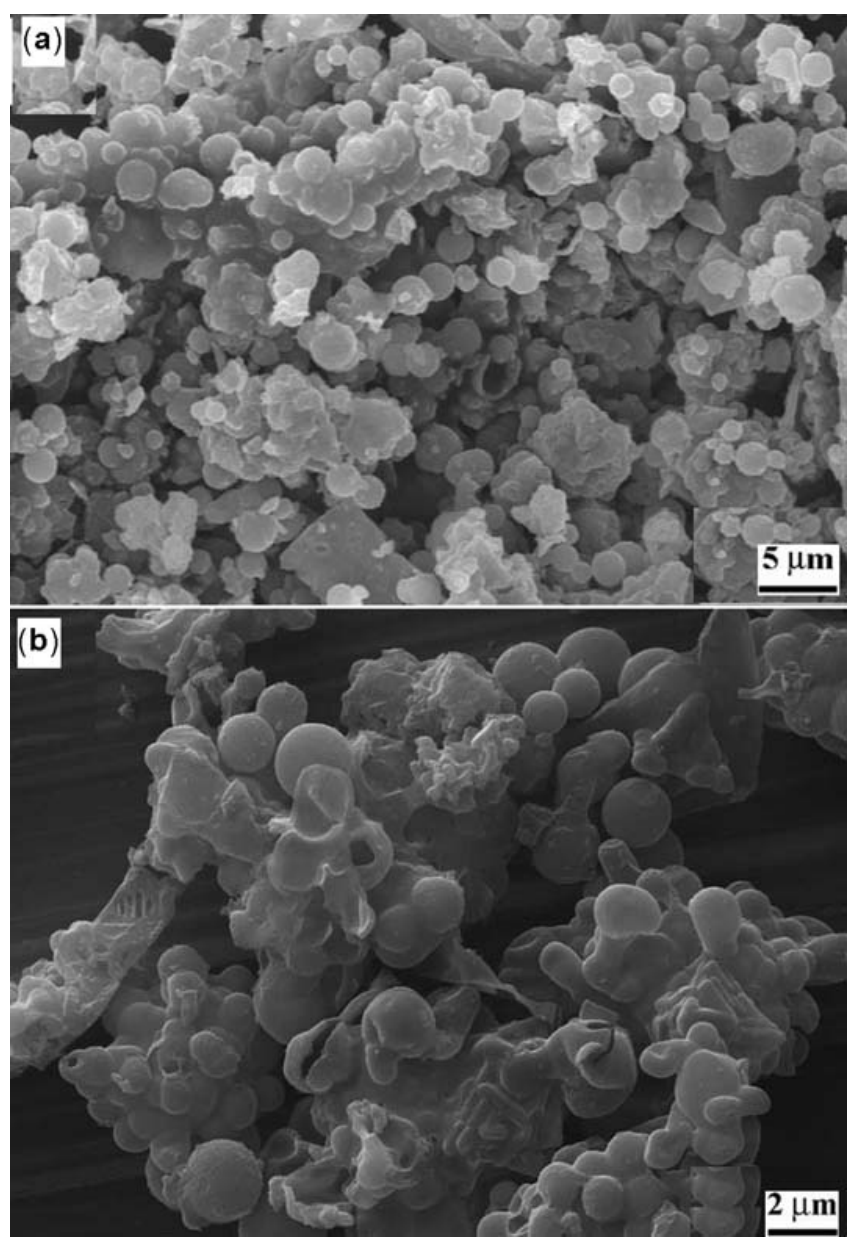

Figure 3. SEM images of (a) the large scale of carbon spheres and (b) the broken hollow carbon spheres. 

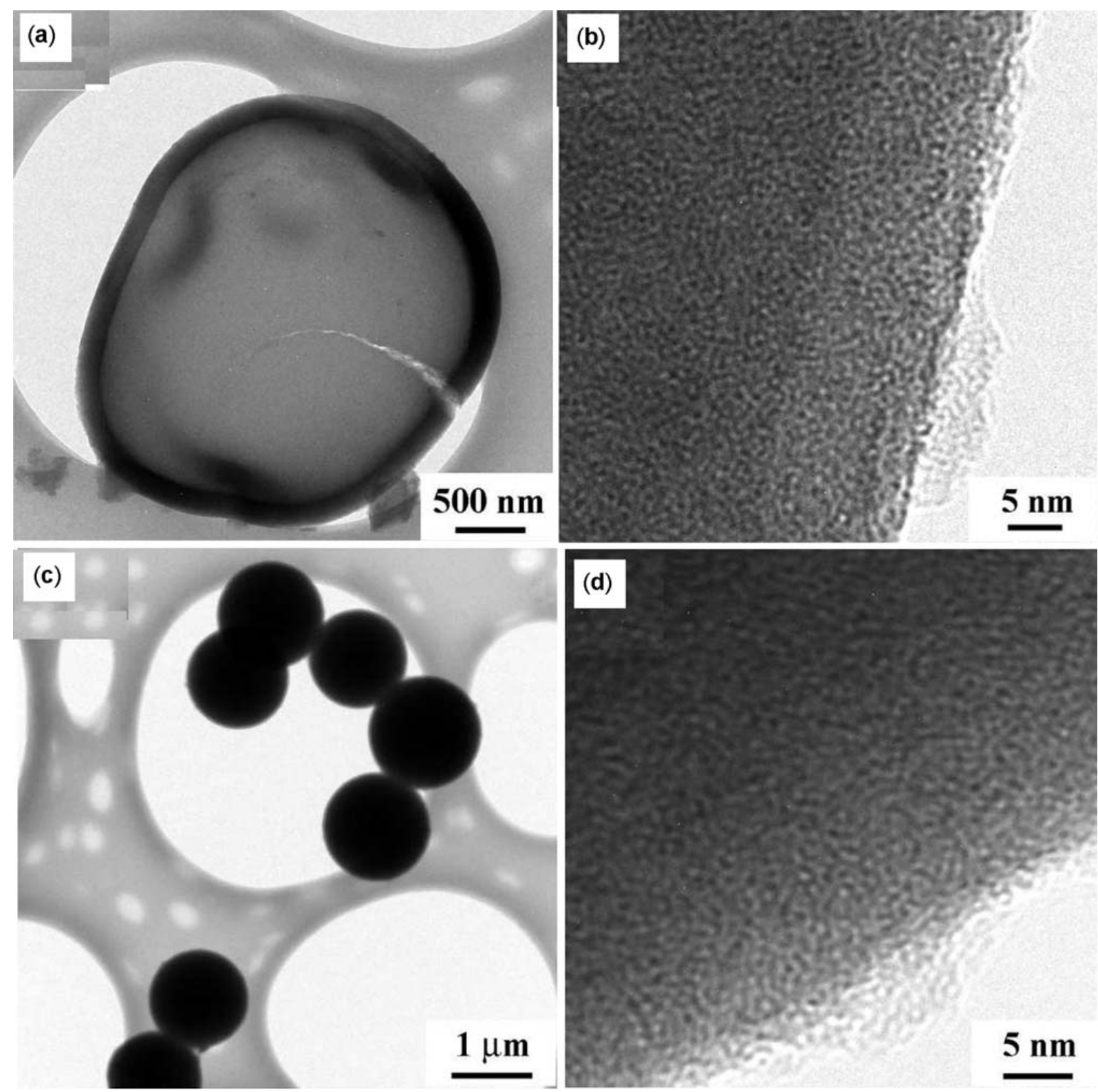

Figure 4. TEM images of (a) one shrivelled HCS, (c) some SCSs and HRTEM images of (b) HCS and (d) SCSs.

sized when ferrocene was used as the only reactant, indicating that ammonium carbonate may play a crucial role in the formation of carbon spheres. The process could be represented as follows: the unstable ammonium carbonate would first decompose into $\mathrm{NH}_{3}, \mathrm{H}_{2} \mathrm{O}$ and $\mathrm{CO}_{2}$ at low temperature. Next, carbon and iron could be produced by the decomposition of ferrocene at about $400^{\circ} \mathrm{C}$ (Hou et al 2002). Then the iron would soon be oxidized by $\mathrm{CO}_{2}$ and $\mathrm{H}_{2} \mathrm{O}$ to form $\mathrm{Fe}_{3} \mathrm{O}_{4}$ (Kaushik and Fruehan 2006), which is detected in the as-prepared product by XRD in figure $1 \mathrm{a}$. And the $\mathrm{Fe}_{3} \mathrm{O}_{4}$ crystals grew larger by consuming the newly generated ions or molecules near the crystal nucleus. At the same time, some carbon feed stock, composed of basic structural units (BSUs), might cover on the sphere- like $\mathrm{Fe}_{3} \mathrm{O}_{4}$ particles to form carbon encapsulated $\mathrm{Fe}_{3} \mathrm{O}_{4}$ and the HCSs could be obtained after acid washing by removal of $\mathrm{Fe}_{3} \mathrm{O}_{4}$ particles. On the other hand, some carbon feed stock would form SCSs to maintain a low surface energy. And the solid/gas interface usually results in a random texture of the sphere due to the random arrangement of the BSUs (Inagaki 1997), leading to the formation of amorphous HCSs and SCSs. Thus, both HCSs and SCSs could be synthesized simultaneously in the process.

However, when $300 \mathrm{mg}$ ammonium carbonate was used instead with other synthesis parameters unchanged, carbon encapsulated six-armed $\mathrm{Fe}_{3} \mathrm{O}_{4}$ would be obtained (supplementary material, figure 5), suggesting that the amount of ammonium carbonate also plays a crucial role on the 


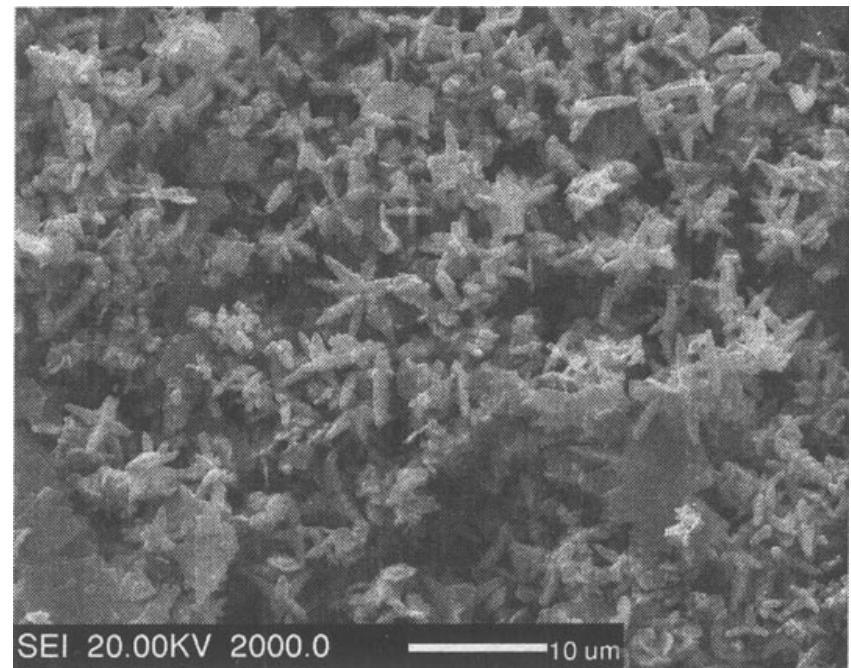

Figure 5. Carbon encapsulated six-armed $\mathrm{Fe}_{3} \mathrm{O}_{4}$ synthesized by the reaction of $100 \mathrm{mg}$ ferrocene and $400 \mathrm{mg}$ ammonium carbonate at $500^{\circ} \mathrm{C}$.

morphology of the product. Therefore, further investigation is needed to better understand the exact effects of ammonium carbonate on the formation of carbon spheres.

\section{Conclusions}

In conclusion, this paper reports a novel method for the synthesis of hollow and solid carbon spheres by the direct reaction of ferrocene and ammonium carbonate in a sealed quartz tube at $500^{\circ} \mathrm{C}$. Ammonium carbonate is crucial for the formation of carbon spheres. The method is efficient and the reactants are inexpensive. Considering that the gas pressure in the reactor is relatively low compared with the solvothermal method, the method may be suitable for large scale preparation in future.

\section{Acknowledgements}

This work is supported by Program for New Century Excellent Talents in University (NCET, No. 04-0327) and Program of Excellent Team in Harbin Institute of Technology.

\section{References}

Antunes E F, Lobo A O, Corat E J, Trava-Airoldi V J, Martin A A and Veríssimo C 2006 Carbon 442202

Bokros J C 1977 Carbon 15353

Hou H Q, Schaper A K, Weller F and Greiner A 2002 Chem. Mater. 143990

Inagaki M 1997 Carbon 35711

Kaushik P and Fruehan R J 2006 Metall. Mater. Trans. B37 715

Lee K T, Jung Y S and Oh S M 2003 J. Am. Chem. Soc. 125 5652

Li L C, Song H H and Chen X H 2006 Carbon 44596

Wang P et al 2007 Mater. Lett. 614854

Wang Y, Su F B, Lee J Y and Zhao X S 2006 Chem. Mater. 18 1347

Wen Z H, Wang Q, Zhang Q and Li J H 2007 Electrochem. Commun. 91867

Wu C Z, Zhu X, Ye L L, OuYang C Z, Hu S Q, Lei L Y and Xie Y 2006 Inorg. Chem. 458543

Yi Z H, Liang Y G, Lei X F, Wang C W and Sun J T 2007 Mater. Lett. 614199

Zhang J, Perez R J and Lavernia E J 1993 J. Mater. Sci. 28 2395

Zhong Z Y, Yin Y D, Gates B and Xia Y N 2000 Adv. Mater. 12206 